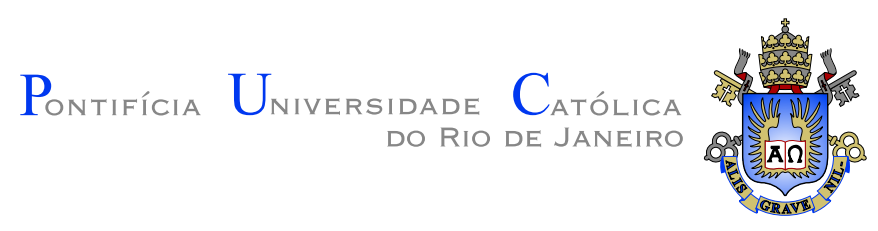

Afonso Paiva Neto

\title{
Uma abordagem lagrangeana para simulação de escoamentos de fluidos viscoplásticos e multifásicos
}

Tese apresentada ao Programa de Pós-graduação em Matemática Aplicada do Departamento de Matemática da PUCRio como requisito parcial para obtenção do título de Doutor em Matemática Aplicada

Orientador: Prof. Geovan Tavares dos Santos

Rio de Janeiro

Agosto de 2007 


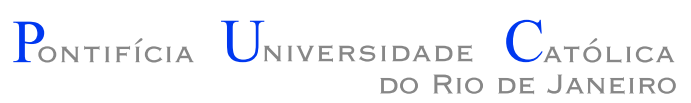

\title{
Afonso Paiva Neto
}

\section{Uma abordagem lagrangeana para simulação de escoamentos de fluidos viscoplásticos e multifásicos}

\begin{abstract}
Tese apresentada ao Programa de Pós-graduação em Matemática Aplicada do Departamento de Matemática do Centro Técnico Científico da PUC-Rio como requisito parcial para obtenção do título de Doutor em Matemática Aplicada. Aprovada pela Comissão Examinadora abaixo assinada.
\end{abstract}

Prof. Geovan Tavares dos Santos

Orientador Departamento de Matemática - PUC-Rio

Prof. Antônio Castelo Filho Instituto de Ciências Matemáticas e da Computação - USP

Prof. Carlos Eduardo Aguiar Instituto de Física - UFRJ

Prof. Luiz Henrique de Figueiredo Instituto Nacional de Matemática Pura e Aplicada - IMPA

Prof. Paulo Roberto de Souza Mendes Departamento de Engenharia Mecânica — PUC-Rio

Prof. Thomas Lewiner Departamento de Matemática - PUC-Rio

Prof. Hélio Côrtes Vieira Lopes

Departamento de Matemática — PUC-Rio

Prof. José Eugenio Leal Coordenador Setorial do Centro Técnico Científico - PUC-Rio 
Todos os direitos reservados. É proibida a reprodução total ou parcial do trabalho sem autorização da universidade, do autor e do orientador.

\section{Afonso Paiva Neto}

Bacharel em Matemática pela Universidade Federal de Uberlândia (2000). Tornou-se Mestre em Matemática pelo Instituto Nacional de Matemática Pura e Aplicada (2003), onde a sua dissertação foi sobre métodos robustos de visualização de atratores estranhos. Durante o doutorado foi membro do laboratório Matmídia do Departamento de Matemática da PUC-Rio, desenvolvendo projetos científicos patrocinados pela Petrobras. Tem experiência na área de Matemática Aplicada, com ênfase em Computação Gráfica e atualmente suas principais áreas de interesse são: modelagem computacional de fenômenos físicos, modelagem geométrica, dinâmica dos fluidos computacional e métodos numéricos auto-validados.

Ficha Catalográfica

Paiva Neto, Afonso

Uma abordagem lagrangeana para simulação de escoamentos de fluidos viscoplásticos e multifásicos / Afonso Paiva Neto; orientador: Geovan Tavares dos Santos. - Rio de Janeiro : PUC-Rio, Departamento de Matemática, 2007.

v., 91 f: il. ; $29,7 \mathrm{~cm}$

1. Tese (doutorado) - Pontifícia Universidade Católica do Rio de Janeiro, Departamento de Matemática.

Inclui referências bibliográficas.

1. Matemática - Tese. 2. Dinâmica de Fluidos Computacional. 3. Smoothed Particle Hydrodynamics. 4. Fluido Viscoplástico. 5. Fluido Multifásico. 6. Análise Numérica. 7. Animação Computacional. 8. Computação Gráfica. I. Santos, Geovan Tavares dos. II. Pontifícia Universidade Católica do Rio de Janeiro. Departamento de Matemática. III. Título. 


\section{Agradecimentos}

Aos meus pais, meu irmão, meus sobrinhos e minha família que souberam lidar com minha ausência e que sem o apoio deles esse sonho não seria realizado.

À Lucilly pela sua paciência, seu amor e também por ser a minha eterna fonte de inspiração.

Ao meu orientador Professor Geovan Tavares pelo apoio durante a realização desse trabalho.

Ao Professor Paulo Roberto Mendes que contribuiu com importantes sugestões que melhoraram a qualidade desse trabalho.

Aos Professores Hélio Lopes, Marcos Craizer, Sinésio Pesco e Thomas Lewiner, os quais compartilharam grandes idéias e boas gargalhadas durante esse tempo.

Aos meus amigos Etereldes (Manjator), Fabiano (Véia Murrinha), Thiago (MochI) e Rener (BiGodz) que ao longo desses anos se tornaram meus irmãos, a minha outra família.

Aos meus colegas da PUC-Rio e do IMPA os quais me fizeram sentir o Rio a minha segunda casa, em especial Adelailson, Alex, Aninha, André (Galeguim), André (Galão), Citeli, Fernando (Ferma), Francisco (Fio), LG, Luís (Lubalf), Marquinhos, Rodrigo (Gaúcho) e Zé Luiz.

Ao pessoal do Departamento de Matemática da PUC-Rio pela ajuda e pelo café de todos os dias, em particular à Ana Cristina, Creuza, Kátia, Orlando e Otávio.

Ao $\mathrm{CNPq}$, à Petrobras e à PUC-Rio, pelos auxílios concedidos, sem os quais esse trabalho não teria sido concluído. 


\section{Resumo}

Paiva Neto, Afonso; Santos, Geovan Tavares dos. Uma abordagem lagrangeana para simulação de escoamentos de fluidos viscoplásticos e multifásicos. Rio de Janeiro, 2007. 91p. Tese de Doutorado - Departamento de Matemática, Pontifícia Universidade Católica do Rio de Janeiro.

Na última década em Computação Gráfica, foram desenvolvidas várias técnicas de simulação do comportamento de objetos como corpos rígidos, água, fumaça, cabelo e tecido. Enquanto essas técnicas se concentram em modelos físicos simplificados através de fluidos newtonianos monofásicos ou de sólidos ideais, propomos aqui novas técnicas de simulação de fluidos viscoplásticos e multifásicos baseadas numa abordagem lagrangeana da equação de quantidade de movimento. Essas técnicas consistem na discretização do fluido através de um sistema de partículas ao invés dos tradicionais métodos baseados em malhas. A simulação computacional da dinâmica de fluidos é feita utilizando o método numérico conhecido como SPH (Smoothed Particle Hydrodynamics). Nessa tese, representamos um material viscoplástico como um fluido não-newtoniano que varia entre os estados sólido e líquido, e de alta para baixa viscosidade dependendo de uma força externa aplicada ao material ou de sua própria temperatura. A simulação de fluidos multifásicos é realizada através de um método híbrido malha-partícula a fim de garantir a incompressibilidade no método SPH.

\section{Palavras-chave}

Dinâmica de Fluidos Computacional. S Smoothed Particle Hydrodynamics. Fluido Viscoplástico. Fluido Multifásico. Análise Numérica. Animação Computacional. Computação Gráfica. 


\section{Abstract}

Paiva Neto, Afonso; Santos, Geovan Tavares dos. A lagrangian approach for simulating viscoplastic and multiphase fluids. Rio de Janeiro, 2007. 91p. PhD Thesis - Departament of Mathematics, Pontifícia Universidade Católica do Rio de Janeiro.

In the last decade in Computer Graphics, they were development many techniques to simulate the behavior of objects such as rigid bodies, water, smoke, hair and cloth. While these techniques focus in simplified physical models through monophase fluids or ideal solids, we propose new simulation techniques of viscoplastic and multiphase fluids based on a lagrangian approach of momentum equation. These techniques consist in the fluid discretization through particles system instead of traditional grid-based methods. The computational fluid dynamics is performed using the method called SPH (Smoothed Particle Hydrodynamics). In this thesis, we represent a viscoplastic material like a non-newtonian fluid that it varies between solid and liquid states, and from low to high viscosity depending on the external force applied on material or its own temperature. The simulation of multiphase fluids is made using a hybrid method grid-particle to guarantee the incompressibility of SPH method.

\section{Keywords}

Computational Fluid Dynamics. Smoothed Particle Hydrodynamics. Viscoplastic Fluid. Multiphase Fluid. Numerical Analysis. Computer Animation. Computer Graphics. 


\section{Sumário}

1 Introdução $\quad 13$

1.1 Trabalhos anteriores 14

$\begin{array}{ll}1.2 \text { Contribuições } & 17\end{array}$

2 Dinâmica dos fluidos clássica $r$

2.1 Formulação lagrangeana 20

2.2 Fluidos não-newtonianos 21

3 Dinâmica dos fluidos computacional $\quad 22$

3.1 Métodos eulerianos 22

3.2 Métodos lagrangeanos 24

3.3 Métodos híbridos 25

4 Dinâmica dos fluidos usando sistema de partículas $\quad 27$

4.1 Smoothed Particle Hydrodynamics (SPH) 27

4.2 Equações de Navier-Stokes usando SPH 33

5 Implementação SPH $\quad 36$

5.1 Busca de partículas vizinhas 36

5.2 Integração numérica temporal 39

5.3 Condição de fronteira 41

5.4 Representação implícita da superfície livre 42

5.5 Exemplo numérico 45

$6 \quad$ Fluidos viscoplásticos $\quad 49$

6.1 Fluido Newtoniano Generalizado 50

6.2 Transição de fase $\quad 51$

6.3 Condição de fronteira 54

6.4 Aspectos numéricos $\quad 59$

6.5 Implementação 60

6.6 Resultados 62

$\begin{array}{lll}7 & \text { Fluidos multifásicos incompressíveis } & \mathbf{7 0}\end{array}$

$\begin{array}{lll}7.1 & \text { Formulação SPH para fluidos multifásicos } & 71\end{array}$

7.2 Incompressibilidade SPH através de um grid 71

7.3 Implementação $\quad 74$

$\begin{array}{lll}7.4 & \text { Resultados } & 79\end{array}$

8 Conclusão e trabalhos futuros $\quad 83$

$\begin{array}{lr}\text { Referências Bibliográficas } & 85\end{array}$ 


\section{Lista de figuras}

1.1 Exemplos de materiais viscoplásticos que sofrem deformação plástica ou que derretem.

1.2 Fluido multifásico: hidrodinâmica de uma bolha de ar dentro da água. À esquerda, uma imagem de uma simulação real e a direita uma imagem de uma simulação numérica com o método híbrido grid-partícula proposto nessa tese.

2.1 Modelos de um volume de controle finito de fluido $d V$ : (a) Volume de controle fixo no espaço com o escoamento do fluido passando através ele. (b) Volume de controle movendo ao longo do escoamento com velocidade $\mathbf{v}$ igual à velocidade local do fluxo naquele ponto.

3.1 (a) Discretização euleriana com campo de velocidade $\mathbf{v}(\mathbf{x})$ nos vértices $\mathbf{x}$ do grid. A região em azul representa o fluido. Representação lagrangeana: (b) discretização utilizando uma malha com campo de velocidade $\mathbf{v}(\mathbf{x})$ nos vértices $\mathbf{x}$ da malha. (c) discretização através de um sistema de partículas com campo de velocidade $\mathbf{v}(\mathbf{p})$ nas partículas $\mathbf{p}$ do sistema.

4.1 Função núcleo: partículas fora do raio de influência $\kappa h$ são descartadas. Em destaque, o gráfico do núcleo 1D e de suas derivadas de primeira e segunda ordem.

5.1 Uma visão geral do ciclo de simulação de um sistema SPH.

5.2 Busca das partículas vizinhas utilizando um grid uniforme bidimensional com o espaçamento sendo o raio de influência $\kappa h$. A região amarela representa as células do grid onde será realizada a busca.

5.3 Estrutura de árvore utilizada no algoritmo de busca e a subdivisão hierárquica do espaço bidimensional. A busca é realizada através de um teste de intersecção do cubo envolvente da partícula $i$ (região em amarelo) com as células representadas pelos nós da árvore.

5.4 Esquema de integração leap-frog: posição $\mathbf{x}_{i}$ e velocidade $\mathbf{v}_{i}$ numa partícula $i$ são avaliadas de maneira intercalada em relação ao tempo $t$.

5.5 As partículas fantasmas (vermelho) são utilizadas para simular as condições de fronteira sólidas, evitando que as partículas de fluido (azul) escapem do domínio do problema.

5.6 Tabela do algoritmo de Marching Cubes (MC) que codifica a triangulação de cada cubo do grid dependendo da configuração dos sinais em seus vértices.

5.7 Superfície implícita de duas esferas próximas uma da outra. À esquerda a superfície é gerada através do algoritmo de MC original. À direita a superfície é gerada utilizando o algoritmo de MC com garantias topológicas. 
5.8 Visualização da superfície implícita extraída do modelo Stanford Bunny constituído de partículas.

5.9 Triangulação adaptativa da isosuperfície do torus utilizando um algoritmo robusto de DMC. As regiões em verde representam as regiões da superfície que possuem garantias topológicas e geométricas, enquanto as regiões com ambigüidades (vermelho) são resolvidas com um número pequeno de refinamentos.

5.10 Configuração inicial do problema.

5.11 Simulação SPH da quebra de uma barragem utilizando $10^{4}$ partículas. 48

6.1 Modelo de Fluido Newtoniano Generalizado: o mapa de cores representa a viscosidade de cada partícula, variando de baixa (azul) para alta viscosidade (vermelho). Note o salto da viscosidade criado pela força aplicada pela mão, quanto maior a força menor será a viscosidade do fluido.

6.2 Comportamento viscoplástico do fluido.

6.3 Variações da viscosidade de um fluido viscoplástico inicializado numa esfera com 1200 partículas. Acima, reduzimos a viscosidade do fluido usando $J=15$ e abaixo aumentamos a viscosidade com $J=150$.

6.4 Temperatura das 10188 partículas do modelo Stanford Bunny: as regiões em azul escuro estão abaixo do ponto de fusão, e assim permanecem sólidas.

6.5 Escoamento de lava no Pão de Açúcar. A simulação da lava é feita utilizando 10137 partículas.

6.7 Simulação de um escoamento de lava após 1910 iterações com colisão suave (esquerda) e com colisão não-escorregadia (direita), com os mesmo parâmetros: 545 partículas e 9566 triângulos na fronteira. O mapa de cores representa a densidade de cada partícula. A colisão suave faz a lava deslizar mais rapidamente.

6.8 O teste de colisão realiza a busca de triângulos nas células do grid através do algoritmo de linhas de Bresenham.

6.10 Intersecção esfera $\times$ segmento de reta.

6.11 Simulação de um escoamento de lava após 1910 iterações, sem XSPH (esquerda) e com a correção XSPH (direita), com a mesma configuração da Figura 6.7. A simulação explode sem XSPH, devido à distância arbitrariamente pequena entre as partículas.

6.12 Ciclo de simulação de um sistema SPH para fluidos não-newtonianos. 61

6.13 Derretimento do modelo Gargoyle totalmente líquido usando 6976 partículas: o mapa de cores representa a temperatura de cada partícula. A estabilidade do método preserva a forma do objeto sem uma representação explícita da malha mesmo após várias iterações.

6.14 Superfície chair modelada como um material viscoplástico com 7000 partículas, interagindo com um objeto sólido complexo representado pelo esqueleto de uma mão com 2351 triângulos. 
6.15 Colisão de uma esfera metálica contra uma parede plástica (verde) constituída por 5000 partículas. A simulação é vista ao mesmo tempo em duas posições diferentes: vista lateral (esquerda) e vista frontal (direta).

6.16 Evolução da superfície livre da simulação de derretimento do modelo Stanford Bunny com 10188 partículas, iniciando frio na base e quente no topo do modelo.

6.17 Derretimento das letras "CGF 2007" usando 10773 partículas, iniciando frio na base e quente no topo do modelo. A adaptatividade do passo de tempo permite uma simulação mais precisa com poucas iterações.

6.18 Escoamento de lava num terreno virtual de 1547 triângulos, com 4900 partículas.

7.1 Os vetores associados à $P$ e $b$ seguem a ordenação natural do grid 2D. 73

7.2 Ciclo de simulação do método híbrido grid-partícula: em azul são as etapas que utilizam $\mathrm{SPH}$ e em verde as etapas que utilizam o grid da pressão.

7.3 Configuração do método híbrido grid-partícula. A dimensão do grid de pressão é a mesma que a do grid utilizado na busca de partículas vizinhas.

7.4 Condição de Neumann num grid $(L+2) \times(M+2)$ : as setas indicam os valores de $P$ copiados para os vértices da fronteira.

7.5 A pressão na partícula $i$ é obtida através de uma interpolação dos vértices de $P$ (verde).

7.6 Gráfico do núcleo quadrático e de sua derivada de primeira ordem.

7.7 Instabilidade de Rayleigh-Taylor formada entre dois fluidos estratificados utilizando 6000 partículas: um mais denso (azul) e um menos denso (cinza). Da esquerda para direita, as imagens acima mostram a evolução da instabilidade nos instantes: $0.0 \mathrm{~s}, 0.8 \mathrm{~s}, 1.2 \mathrm{~s}$, $1.7 \mathrm{~s}$ e $2.1 \mathrm{~s}$.

7.8 Evolução da altura da instabilidade de Rayleigh-Taylor: comparação da simulação numérica com a solução analítica.

7.9 Campo vetorial da velocidade: (a) As cores representam a magnitude da velocidade do fluido, variando de baixa velocidade (azul) para alta (vermelho). (b) No campo de fase as cores representam a variação do coseno do ângulo formado pelo vetor velocidade e o eixo das abscissas indo de -1 (vermelho) até 1 (azul). (c) Os vórtices são melhores identificados através das linhas de fluxos.

7.10 Movimento hidrodinâmico de uma gota de um fluido mais leve (azul) no interior de um fluido mais pesado (cinza) utilizando 6000 partículas.

7.11 Evolução da altura de uma gota: comparação da simulação numérica com a solução analítica. 


\section{Lista de tabelas}

6.1 Atributos da partícula. 61

6.2 Tempo médio de iteração em cada simulação executadas num computador com processador Pentium $4-2.4 \mathrm{GHz}$ e com $2 \mathrm{~Gb}$ de RAM. 
You have entered the Twilight Zone

Beyond this world strange things are known Use the key, unlock the door

See what your fate might have in store...

Come explore your dreams' creation

Enter this world of imagination...

Rush, The Twilight Zone. 